\section{La investigación en salud y la evaluación de tecnologías sanitarias en Chile}

\author{
MANUEL ANTONIO ESPINOZA ${ }^{1,2, a}$, BÁLTICA CABIESES ${ }^{3,4, b}$, \\ GUILLERMO PARAJE ${ }^{5, c}$
}

\section{Health research and Health Technology Assessment in Chile}

Health research is considered an essential element for the improvement of population health and it has been recommended that a share of the national health budget should be allocated to develop this field. Chile has undertaken efforts in the last decades in order to improve the governmental structure created to promote the development of health research, which has increased human resources and funding opportunities. On the other hand, the sustained economic growth of Chile in the last decades suggests that the health expenditure will maintain its increasing trend in the following years. This additional funding could be used to improve coverage of current activities performed in the health system, but also to address the incorporation of new strategies. More recently, health technology assessment (HTA) has been proposed as a process to support decisions about allocation of resources based on scientific evidence. This paper examines the relationship between the development of health research and the HTA process. First, it presents a brief diagnosis of the situation of health research in Chile. Second, it reviews the conceptual basis and the methods that account for the relationship between a HTA process and the development of health research. In particular, it emphasizes the relevance of identifying information gaps where funding additional research can be considered a good use of public resources. Finally, it discusses the challenges and possible courses of action that Chile could take in order to guarantee the continuous improvement of an articulated structure for health research and HTA.

(Rev Med Chile 2014; 142 (S1): S 39-44)

Key words: Health services research; Health technologies; Chile.
'Subdepartamento de Estudios y Evaluación de Tecnologías Sanitarias, Departamento de Asuntos Científicos, Instituto de Salud Pública, Santiago, Chile.

2Departamento de Salud Pública, Facultad de Medicina, Pontificia Universidad Católica de Chile, Santiago, Chile.

${ }^{3}$ Facultad de Medicina,

Universidad del Desarrollo Clínica Alemana, Santiago, Chile.

${ }^{4}$ Department of Health Sciences, University of York, UK.

${ }^{5}$ Escuela de Negocios, Universidad Adolfo Ibáñez.

a Médico, PhD en Economía ( $U$.

York, UK), MSc en Economía de la Salud (U. York), Magíster en Bioestadística (U. de Chile), Magíster en Epidemiología (UC).

${ }^{b}$ Enfermera-Matrona, PhD en Epidemiología Social (U. York, UK), Magíster en Epidemiología (UC).

'Economista, PhD en Economía (U. Cambridge, UK), MPhil en Economía (U. Cambridge, UK), MA en Economía (Georgetown U., USA).

Fuentes de financiamiento: Este artículo no contó con ningún tipo de apoyo financiero. Los autores declaran no poseer conflictos de intereses que puedan sesgar los contenidos expresados en el artículo.

Correspondencia a:

Dr. Manuel Antonio Espinoza S., MSc PhD. Departamento de Salud Pública, Pontificia Universidad Católica de Chile. Marcoleta 434, Santiago, Chile. Fono: 23546807.
L a Comisión de Investigación en Salud para el Desarrollo ha recomendado en 1990 que el $2 \%$ del presupuesto nacional de salud sea destinado a investigación en salud, meta que muchos países en Latinoamérica no han logrado alcanzar ${ }^{1}$. Otros índices internacionales cifran como objetivo el destinar a lo menos $1 \%$ del producto geográfico bruto a ciencia y tecnología, lo que no ha sido nunca alcanzado por países latinoamericanos ${ }^{2}$. Mientras países desarrollados llegan a cifras de 2,7\%, en Latinoamérica estos gastos fluctúan entre 0,24 y $0,75 \%$. En Chile se ha estimado que los aportes de diferentes órganos financieros para el año 2000 alcanzan entre 0,6 y $0,8 \%{ }^{3}$.

La idea central reportada en la literatura disponible en las últimas dos décadas es que Chile debiera continuar su inversión en investigación en salud como una prioridad ${ }^{3,4}$, algo que se ha reflejado en importantes esfuerzos durante los últimos 10 años. Destaca por ejemplo la creación del Fondo Nacional de Investigación en Salud (FONIS) el año 2003, que hasta diciembre del 
año 2012 había realizado 9 concursos abiertos. A esto se le suman otros fondos nacionales que habitualmente destinan fondos a investigación en salud como FONDEF y FONDECYT.

Por otro lado, la implementación de la Evaluación de Tecnologías en Salud (ETESA) en los sistemas de salud no sólo ha sido motivada por la necesidad de contar con un proceso legítimo de decisiones sobre cobertura de intervenciones en salud basadas en evidencia científica sino también para mejorar la eficiencia en la identificación, selección y evaluación de aquellos problemas de salud que deben ser atendidos antes que otros. La implementación de ETESA está ligada, por un lado al desarrollo de capacidades profesionales para conducir investigación relevante para la toma de decisiones, y por otro lado, a la identificación de vacíos de información y priorización de problemas sanitarios que deben ser atendidos en el corto plazo.

El presente artículo tiene por objetivo examinar la relación que existe entre ETESA e investigación en salud, en particular cómo la implementación de ETESA pudiese impactar el desarrollo de la investigación en salud en Chile. En la primera sección se describe un breve diagnóstico de situación de la investigación en salud en Chile. En la segunda sección se examina por qué ETESA debiese estar articulada con la institución que define la asignación de recursos para investigación sanitaria y los instrumentos que pueden ser utilizados para este fin. Finalmente, se discuten los desafíos que tiene Chile en esta materia, elaborando algunas propuestas que pueden ser de utilidad en la implementación de ETESA.

\section{Investigación en salud en Latinoamérica y Chile}

Un estudio de la Organización Panamericana de la Salud (OPS) de los años 90 identificó como características comunes de Chile y algunos de los países de Latinoamérica el pobre desarrollo de la investigación y su sustento tecnológico, así como una escasa multi o trans-disciplinariedad en investigación en salud ${ }^{5,6}$. Un estudio similar al de la OPS, publicado el $2010^{4}$, describió los subsistemas públicos de los Sistemas Nacionales de Investigación en Salud (SNIS) en cinco países de América Latina (Argentina, Bolivia, Chile,
Paraguay y Uruguay), con énfasis en los tipos de arreglos institucionales que se observan en cada país para promover, desarrollar y sostener sus SNIS, así como en los mecanismos explícitos o implícitos de priorización de proyectos de investigación en salud. Los resultados del estudio permiten reflejar algunos avances en investigación en salud en la región en la última década, pero también reconocen algunas limitaciones y desafíos pendientes. Por ejemplo, numerosas interrogantes surgen acerca de la capacidad de estos países para adaptar y generar nuevos conocimientos. También se identifica una escasa investigación sobre condicionantes sociales, económicos y culturales o sobre servicios y sistemas de salud. Los autores señalan que es necesario establecer explícitamente las prioridades en la agenda de investigación en salud, en consenso con las partes interesadas, así como incorporar mecanismos de monitoreo y seguimiento por temas y áreas de estudio.

En Chile, la gestión y la asignación de los recursos públicos concursables para investigación en salud están a cargo de la Comisión Nacional de Investigación Científica y Tecnológica (CONICYT), que contiene al Fondo de Fomento al Desarrollo Científico y Tecnológico (FONDEF). Al interior del FONDEF se encuentra el Fondo Nacional de Investigación en Salud (FONIS), que cuenta con financiamiento combinado del Ministerio de Salud y del Ministerio de Educación ${ }^{4,7}$. Así, Chile destaca por presentar organismos autónomos que implementan programas regulares, con reglamentos y objetivos definidos y relativamente permanentes en el tiempo, brindando cierta estabilidad a la participación pública en el financiamiento de la investigación ${ }^{4}$. Aunque la gestión de CONICYT es la más ampliamente reconocida, la autoridad también financia investigación directamente con el presupuesto de sus departamentos en aquellos temas que les son prioritarios. Otros organismos públicos relevantes, tales como diferentes departamentos al interior del Ministerio de Salud de Chile (MINSAL), el Instituto de Salud Pública de Chile (ISP) y la Superintendencia de salud; también gestionan el financiamiento en investigación en salud.

En términos de la estructura general de la investigación en salud en Chile, destacan cuatro elementos: 1) Chile presenta un importante gasto público en proyectos de investigación cuando se compara con otros países de Latinoamérica (Figura 1);2) Las principales líneas de investigación 
son, en orden decreciente la biomédica, la clínica y luego salud pública; 3) Si bien la investigación en salud en Chile es importante como para destinar fondos a ella de manera estable (aunque por debajo de la meta del $2 \%$ sugerida por OMS), aun no se generan pautas explícitas acerca de cuáles son los temas prioritarios que se requieren investigar ${ }^{4}$; y 4) Existe una concentración inusual de investigadores principales en la Región Metropolitana y una baja proporción de mujeres ${ }^{7}$.

Si bien la mayoría de los fondos de investigación en salud en Chile están enfocados en ciencias básicas, existe un fondo pequeño de investigación aplicada en salud que ha aportado a reducir moderadamente esta brecha, el ya mencionado FONIS. Un estudio del año $2007^{8}$ indica que la mayoría de los estudios postulados y adjudicados en los tres primeros concursos de FONIS estuvieron dedicados a responder problemáticas de salud pública (48\%) luego problemas clínicos (36\%) y de gestión de servicios (18\%). La mayoría de los investigadores que se adjudican este fondo provienen de Universidades del país, públicas y privadas. Los estudios de salud pública son en su mayoría de carácter epidemiológico, los clínicos enfocados a proponer nuevos tratamientos (y luego prevención o diagnostico), y los de "gestión de servicios" que incluyen estudios de evaluación de impacto y evaluaciones económicas en salud, siendo éstos últimos los menos frecuentes. La mayoría de los proyectos financiados responde a algún objetivo sanitario de la década y la mayoría de ellos fueron cuantitativos. La mayor parte de los proyectos adjudicados provienen de la Región Metropolitana, seguida por la VIII, IX, V y la VII, y la proporción de mujeres que obtienen este fondo ha aumentado progresivamente a lo largo del tiempo.

\section{ETESA e investigación: ¿dónde está la unión?}

Las ETESA emergen primero como un método y luego como un proceso de identificación, selección y evaluación de información que cumpla con un objetivo central: ser capaz de orientar y dar soporte a decisiones sobre incorporación de intervenciones al arsenal diagnóstico, terapéutico y preventivo de un sistema de salud fundado en las bases de cobertura universal y protección financiera de sus ciudadanos. Debido a que el proceso se establece para apoyar la toma de decisiones, la representación y caracterización empírica de la incertidumbre que rodea la evidencia científica resulta esencial9.

La incertidumbre implica que existe una probabilidad de que la decisión que se tome en políticas de salud sea incorrecta. Luego, las decisiones incorrectas determinan consecuencias indeseadas

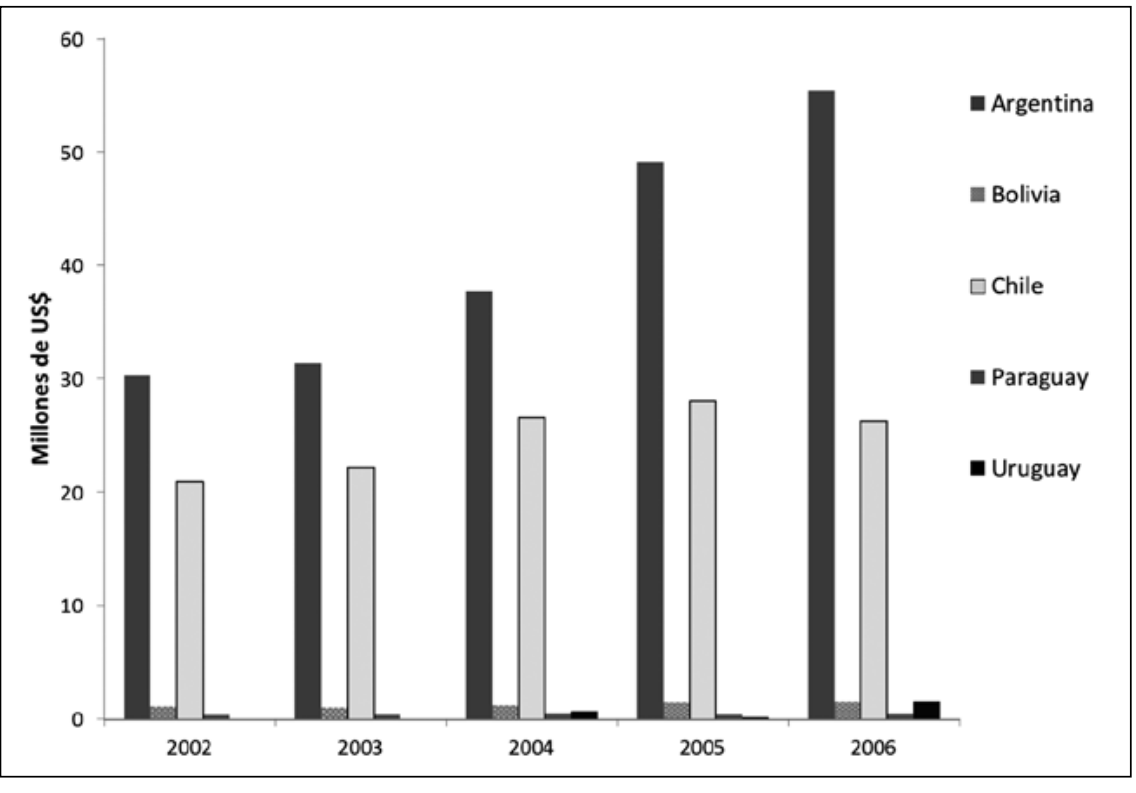

Figura 1. Distribución del financiamiento público para la investigación en salud, por áreas de estudio, en 5 países de América Latina, 2006 (en miles de US\$ ajustados según paridad de poder adquisitivo). Reproducido con autorización de Maceira y cols $(2010)^{4}$. 
que pueden ser cuantificadas como pérdidas en salud. En un contexto de recursos limitados, la estimación de estas consecuencias deben incluir además el impacto en términos del costo de oportunidad de una asignación alternativa de dichos recursos ${ }^{10}$. Estos elementos han sido desarrollados en el campo de investigación de operaciones desde mediados del siglo pasado ${ }^{11}$ y aplicados más recientemente en salud ${ }^{12}$. Estos elementos forman parte de un marco analítico llamado "valor de la información", el cual sugiere que el costo de la incertidumbre es equivalente al valor de contar con información perfecta. Es decir, una muestra representativa de la población que sea capaz de llevar a cero la probabilidad de cometer una decisión incorrecta. Este valor se expresa como el valor esperado de la información perfecta (VEIP), métrica que puede ser expresada en términos monetarios o de salud y representa el límite superior de lo que el sistema debiese estar dispuesto a pagar por solucionar la incertidumbre ${ }^{13}$. Más recientemente, estos métodos han sido extendidos al análisis de heterogeneidad en el contexto de análisis de costo-efectividad ${ }^{14}$.

En este marco conceptual el VEIP se entiende como una condición necesaria para la decisión de financiar o no una futura investigación y si ésta representa un buen uso de los recursos. Sin embargo, no ofrece más información que oriente al tipo de investigación que debe ser realizada. En este contexto, resulta importante evaluar qué parámetros (ej. eficacia, costos, utilidades) son los que están asociados a mayor incertidumbre y por lo tanto ofrecen una mejor oportunidad donde concentrar recursos de investigación ${ }^{15}$. La estimación del valor esperado de la información perfecta para parámetros (VEIPP) permite cuantificar este valor, ofreciendo una magnitud que puede ser utilizada para priorización de estudios ${ }^{16}$. La literatura ofrece varios ejemplos que han estimado estas métricas donde el lector puede profundizar el entendimiento de estos métodos y sus implicancias ${ }^{12,17,18}$.

Un tercer elemento esencial en la definición de futura investigación de investigación es el tamaño muestral del estudio. La inferencia estadística clásica plantea que este tamaño es función de 4 elementos: la confianza, la potencia, la precisión y la magnitud de efecto esperada. Aunque este marco conceptual está ampliamente validado, no incluye consideraciones de eficiencia, un elemento fundamental desde la perspectiva de quien asigna recursos para investigación sanitaria. El marco analítico de valor de la información ha creado herramientas para enfrentar este problema. El valor esperado de la unidad muestral ó EVSI (del inglés expected value of sampling information), representa el valor (en términos monetarios o de salud) de una unidad adicional muestreada para efectos de resolver la incertidumbre actual en el sistema de salud ${ }^{19}$. La diferencia entre el valor ofrecido por un estudio de tamaño $\mathrm{n}$ y su costo es el llamado Beneficio Neto Esperado del Muestreo (BNEM), el cual puede ser estimado para distintos tamaños muestrales ( $\mathrm{n})$. El $\mathrm{n}$ donde el BNEM es máximo debiese ser considerado como el tamaño muestral apropiado óptimo para resolver la incertidumbre del sistema de salud ${ }^{20}$. Esta visión del cálculo de tamaño muestral contrasta fuertemente con la metodología que utiliza la inferencia estadística clásica descrita arriba. Sin embargo, resulta más racional para efectos de asignación de recursos en cuanto incluye una consideración de costo oportunidad que está ausente del marco estadístico clásico.

\section{Discusión}

Dado el grado de desarrollo de Chile y su estructura demográfica actual, es probable que el gasto en salud siga aumentado en términos absolutos. En este contexto, se hace fundamental que el país cuente con una estructura que permita dar soporte a las decisiones de cómo gastar el dinero destinado a nuevas intervenciones sanitarias. Debido a que estas tecnologías, procedimientos o políticas recientemente incorporadas tienen asociado grados variables de incertidumbre, la autoridad sanitaria está obligada a preguntarse si el uso de recursos en investigación representa un buen uso de los recursos sanitarios. Adicionalmente, en aquellos casos donde los vacíos de información son completos y no existen incentivos para el desarrollo de investigación privada, es el estado quien debe hacerse cargo y promover la generación de información en estas áreas.

La investigación en salud que se desarrolla en Chile en la actualidad está fundamentalmente alojada en centros académicos y en menor medida en la estructura del sistema de salud y el sector privado. Si bien no existe una estimación precisa del financiamiento disponible para investigación 
sanitaria en Chile, éste parece corresponder a una proporción bastante menor del $1 \%$, lejos de las recomendaciones internacionales. Adicionalmente, una parte importante de estos recursos son puestos a disposición de los intereses de los investigadores, los cuales en general están alineados con las prioridades de la autoridad sanitaria, pero no es algo que se evalúe formalmente en el país. Sería útil definir y delimitar en Chile cuáles fondos permiten el desarrollo de estudios de generación de nuevo conocimiento sin que se enmarquen dentro de los objetivos sanitarios del país, versus aquellos fondos que intentan resolver prioridades de salud pública nacional. Al mismo tiempo, estas prioridades no son siempre explícitas lo cual no da garantía del retorno de la inversión. Debido a los limitados recursos que siguen esta vía es posible que la pérdida no sea significativa. No obstante, en un escenario de aumento del presupuesto de investigación la identificación de vacíos de información y selección debe asegurar eficiencia del uso de recursos en investigación.

Es importante agregar que la implementación de un proceso de ETESA ofrece una oportunidad para mejorar la eficiencia en la definición y asignación de recursos para investigación aplicada en salud en un marco translacional del conocimiento ${ }^{21}$ y potencialmente para reducir las desigualdades en salud ${ }^{22}$. A lo largo de la ETESA existe la posibilidad de caracterizar la incertidumbre, lo cual permite identificar aquellas áreas donde el gasto en investigación ofrece mayor valor. Los métodos de valor de la información han sido aplicados en salud para este fin por NICE en Inglaterra y han sido reconocidos por su aporte a la entrega de nueva información en el proceso de toma de decisión nacional ${ }^{12}$ y más recientemente ha despertado interés de agencias en Estados Unidos ${ }^{23}$.

Por último, la instalación de un proceso de ETESA va de la mano con una reformulación de los fondos públicos y la forma de definir y articular las prioridades de investigación en salud en Chile ${ }^{24,25}$. Este país cuenta con el capital humano y financiero para desarrollar mecanismos políticoinstitucionales formales y transparentes que permitan evaluar las decisiones sobre qué debe ser investigado en Chile, cómo debe generarse dicha evidencia y cuál es el retorno concreto que ese nuevo conocimiento le ofrece al país si proviene de fondos públicos.

\section{Referencias}

1. Commission on Health Research for Development, Health Research: Essential link to equity in Development 1990: Oxford University Press.

2. Dellacasa C. Comisión Nacional de Investigación Científica y Tecnológica CONICYT. 1998; Santiago.

3. Stockins B. [Health research in Chile]. Rev Med Chile 2000; 128 (12): 1389-95.

4. Maceira D, Paraje G, Aramayo F, Masi SD, Sánchez D. [Public financing of health research in five Latin American countries]. Rev Panam Salud Publica 2010; 27 (6): 442-51.

5. Pellegrini F. Science for health: notes on the organization of the scientific activity for the development of health in Latin America and the Caribbean. Rev Panam Salud Pública 2000; 5: 345-9.

6. Pellegrini F. Introduccion a la investigacion en salud en America Latina: estudio de paises seleccionados. Organizacion Panam Salud Pública 1992; 543.

7. Paraje G. [Public financing of health research in Chile]. Rev Med Chile 2010; 138 (1): 36-43.

8. Vidales A. La investigación aplicada en salud en Chile. Rev Chil Salud Pública 2007; 11(3): 158-66.

9. Griffin S, Claxton K, Hawkins N, Sculpher M. Probabilistic analysis and computationally expensive models: Necessary and required? Value Health 2006; 9 (4): 24452.

10. Claxton K, Sculpher M, and Drummond M. A rational framework for decision making by the National Institute For Clinical Excellence (NICE). Lancet 2002; 360 (9334): 711-5.

11. Raiffa H, Schlaifer R. Probability and Statistics for Business Decisions 1959, New York: McGraw-Hill.

12. Claxton K, Sculpher M.J. Using value of information analysis to prioritise health research: some lessons from recent UK experience. Pharmacoeconomics 2006; 24 (11): 1055-68.

13. Claxton K. The irrelevance of inference: a decisionmaking approach to the stochastic evaluation of health care technologies. J Health Econ 1999; 18 (3): 341-64.

14. Espinoza M, Manca A, Claxton K, Sculpher M. The Value of Heterogeneity for Cost-Effectiveness Subgroup Analysis: Theoretical Framework and Application. Value Health 2011; 14 (7): A241-A241.

15. Griffin S, Welton NJ, and Claxton K. Exploring the Research Decision Space: The Expected Value of Information for Sequential Research Designs. Med Decis Making 2010; 30 (2): 155-62.

16. Brennan A, Kharroubi S, O’Hagan A, Chilcott J. Calcu- 
lating partial expected value of perfect information via Monte Carlo sampling algorithms. Med Decis Making 2007; 27 (4): 48-70.

17. Bojke L, Claxton K, Sculpher MJ, Palmer S. Identifying research priorities: the value of information associated with repeat screening for age-related macular degeneration. Med Decis Making 2008; 28 (1): 33-43.

18. Philips Z, Claxton KP, Palmer S, Bojke L, Sculpher MJ. Priority setting for research in health care: an application of value of information analysis to glycoprotein IIb/Illa antagonists in non-ST elevation acute coronary syndrome. Int J Technol Assess Health Care 2006; 22 (3): 379-87.

19. Ades AE, Lu G, Claxton K. Expected value of sample information calculations in medical decision modeling. Med Decis Making 2004; 24 (2): 207-27.

20. Brennan A, Kharroubi SA. Efficient computation of partial expected value of sample information using Bayesian approximation. J Health Econ 2007; 26 (1): 122-48.
21. Cabieses B, Espinoza M. Towards a new framework for health research in Chile: a proposal. Rev Med Chile 2012; 140 (1): 125-7.

22. Cabieses B, Espinoza M. Redistributing health through public health policies in Latin America: fair to whom and fair how? Rev Panam Salud Pública 2012; 32 (5): 387-8.

23. Claxton K, Griffin S, Koffijberg H, McKenna C. Expected health benefits of additional evidence: Principles, methods and application. Centre for Health Economics Discussion Paper 2013; 83.

24. Cabieses B, Espinoza MA. [Translational research and its contribution to the decision making process in health policies]. Rev Peru Med Exp Salud Pública 2011; 28 (2): 288-97.

25. Espinoza M. and Cabieses B. Examining the link between equity-centred health policies, autonomy and decision-making process in low and middle income countries. Br J Med Med Res 2013; 3 (4): 1517-29. 